\title{
Des mutations d'un récepteur couplé à une protéine $G$ sont cause d'anomalies familiales du métabolisme du calcium
}

On connaît déjà un certain nombre de maladies dues à des mutations de récepteurs couplés à des protéines G. Un exemple particulièrement intéressant vient d'en être fourni dans un article, paru le 31-12-93 dans Cell, provenant de Boston, MA, et de Bethesda, MD USA [1]. Il concerne deux maladies qui provoquent des troubles du métabolisme du calcium. L'hypercalcémie hypocalciurique familiale (HHF) reconnaît une hérédité autosomique dominante; elle reste bénigne, sans complications rénales ni hypertension, et ne modifie pas la sécrétion de parathormone par les parathyroïdes malgré l'hypercalcémie (sécrétion inappropriée), comme si la sécrétion d'hormone parathyroïdienne (PTH) n'était pas bien contrôlée par la concentration sanguine de $\mathrm{Ca}^{2+}$. $\mathrm{La}$ même anomalie est sans doute présente dans le rein, incapable d'augmenter la calciurie proportionnellement à l'hypercalcémie. Au contraire, l'hyperparathyroïdie néonatale sévère, d'hérédité récessive, beaucoup plus rare, est généralement létale en l'absence d'une parathyroïdectomie précoce. Le fait que les deux formes puissent coexister dans une même famille a fait penser que l'une est la forme homozygote de l'autre. Brown et al. (Boston, MA) ont identifié récemment, par clonage d'expression dans des ovocytes de xénope, un ADNc d'un récepteur bovin sensible au calcium provenant de cellules parathyroïdiennes [2]. L'ADNc code pour une protéine de $120 \mathrm{kDa}$, dotée, $\mathrm{m} / \mathrm{s} n^{\circ} 4$ vol. 10, avril 94 d'après sa séquence, des sept segments transmembranaires caractéristiques des récepteurs de surface couplés aux protéines G. Dans les parathyroïdes, ce récepteur est selon toute vraisemblance, le "senseur" du $\mathrm{Ca}^{2+}$ circulant, contrôlant la sécrétion de parthormone en fonction de la calcémie via la voie des inositolphosphates. En revanche, on ne connaît pas la fonction du récepteur dans le rein et le cerveau où il est également synthétisé.

Le gène incriminé dans la HHF a été localisé par analyse de liaison sur le chromosome 3 en $3 q 2$ ([3], $\mathrm{m} / \mathrm{s}$ $n^{\circ} 10$, vol. 8, p. 1120). Utilisant à l'origine l'ADNc bovin, Pollak et al [1] ont identifié le gène humain homologue sur le chromosome 3 humain ; ils ont pu analyser la structure du gène humain, qui s'étend sur au moins $20 \mathrm{~kb}$ et compte six exons. Trois familles atteintes de HHF furent examinées, en prenant comme méthode principale une digestion à la RNase. Une anomalie fut détectée dans un seul exon dans chaque famille. On a ainsi identifié trois mutations faux-sens; pour les dénommer, les auteurs ont adopté la numérotation de la protéine bovine, qui compte 1085 acides aminés, bien que, d'après une figure de [1], il semble que la protéine humaine n'en ait que 1079 .

Dans la première famille, trois clones sur huit émanant de l'exon 6 avaient une transition $\mathrm{C} \rightarrow \mathrm{T}$, conduisant à une mutation hétérozygote Arg 796 Trp. Dans les deux autres familles la mutation siégeait dans l'exon 3. Chez un sujet atteint de HHF, trois clones sur sept avaient une transition $G \rightarrow A$, entraînant un changement Arg 186 Glu. Dans la troisième famille une transition $G \rightarrow$ A changeait Glu 298 en Lys. Cette famille comprenant des sujets à symptomatologie HHF, hétérozygotes pour l'anomalie, et un sujet atteint de la forme grave d'hyperparathyroïdie néonatale, qui s'est révélé homozygote, confirmant ainsi que les deux maladies affectaient le même gène. L'absence de telles mutations a été vérifiée chez cinquante témoins. Pour s'assurer que les altérations de séquence étaient responsables de variations de sensibilité au calcium, les auteurs ont effectué des études d'expression dans des ovocytes de xénope. Des mutants d'ARN furent préparés à partir d'ADNc bovin portant la mutation R796W. Les ovocytes furent testés pour leur réponse à des agonistes du récepteur cationique, tels que calcium, gadolinium, ou néomycine. Les préparations d'ARN mutant montrèrent une forte diminution de la réponse $(5 \%$ à $10 \%$ ) (courent $\mathrm{Cl}^{-}$étudié en patch clamp), démontrant les conséquences pathologiques de la mutation.

Les mutations des protéines $G$ et surtout de leurs récepteurs sont nombreuses. Une mini-revue de Cell [4] cite huit maladies dues à une anomalie d'un récepteur, et quatre dues à une protéine $G$ ellemême. Comme cette même minirevue estime à environ 1000 l'ensemble des types de récepteurs

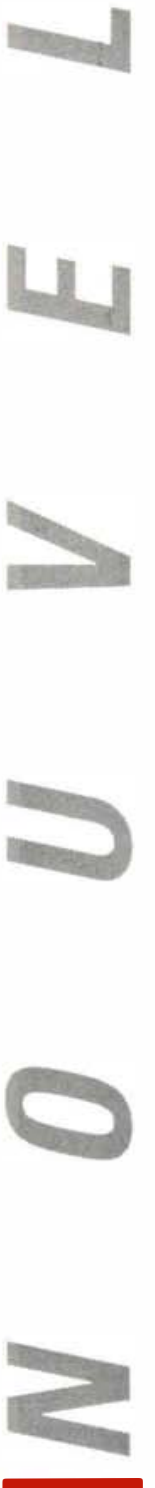


couplés à des protéines $G$, on n'est pas près de voir s'épuiser la liste des maladies dues à leurs anomalies. Un intérêt particulier tient à ce qu'un certain nombre de ces maladies sont dues à l'activation constitutive de ces récepteurs, comme l'a rapporté récemment Vassart dans $m / s[5]$. Tel ne semble pas être le cas du récepteur sensible au calcium, puisque les études d'expression montrent une baisse de la sensibilité. Ces mutations paraissent faire partie de celles qui altèrent la liaison aux agonistes.

Comment interpréter l'effet des mutations observées? C'est d'autant plus difficile que les domaines fonctionnels du récepteur sensible au calcium ne sont pas encore clairement définis. Les deux mutations de l'exon 3 affectent une zone extra- cellulaire, potentiellement impliquée dans la liaison du calcium au voisinage du N-terminal. La mutation 796 siège dans la dernière des trois boucles intracellulaires. Cette région interviendrait dans la transduction du signal, ainsi que dans une séquence de phosphorylation par la protéine kinase C. Il est probable que ces explications ne sont pas définitives, et que la compréhension des mécanismes régulateurs du métabolisme calcique devra encore progresser avant d'avoir une interprétation complète.
I. Pollak MR, Brown EM, Chou YHW, Hebert SC, Marx SJ, Steimann B, Levi T, Seidman CE, Seidman JG. Mutations in the human Ca-sensing receptor gene cause familial hypocalciuric hypercalcemia and neonatal severe hyperparathyroidism. Cell $1993 ; 75$ : 1297-303.

2. Brown EM, Gamba G, Riccardi D, Lombardi M, Butters R, Kif or O, Sun A, Heidiger M, Lytton J, Hebert SC. Cloning, expression and characterization of an extracellular Ca sensing receptor from bovine parathyroid. Nature $1993 ; 366$ : 575-80

3. Chou YHW', Brown EM, Levi T, Crowe G, Atkinson AB, Arnquist HJ, Toss G, El-Hajj Fuleihan G, Seidman JG, Seidman CE. The gene responsible for familial hypocalciuric hypercalcemia maps to chromosome $3 q$ in four unrelated families. Nature Genet 1992; 1 : 295-9.

4. Clapham DE. Mutations in G proteinlinked receptors: novel insights on disease. Cell 1993; 75 : 1237-9.

5. Vassart G. Des maladies provoquées par J.C.D. l'activation constitutive de récepteurs couplés J.P.G. 1421 . 